\title{
Segmental analysis by speckle-tracking echocardiography of the left ventricle response to isoproterenol in male and female mice
}

\author{
Elisabeth Walsh-Wilkinson ${ }^{1}$, Marie Arsenault ${ }^{1}$, Jacques Couet ${ }^{\text {Corresp. } 1}$ \\ ${ }^{1}$ Universite Laval, Groupe de recherche sur les valvulopathies, Centre de recherche de I'Institut universitaire de cardiologie et de pneumologie de \\ Quebec, Quebec, Quebec, Canada \\ Corresponding Author: Jacques Couet \\ Email address: jacques.couet@med.ulaval.ca
}

We studied by conventional and speckle-tracking echocardiography, the response of the left ventricle (LV) to a three-week continuous infusion of Isoproterenol (Iso), a non-specific beta-adrenergic receptor agonist in male and female C57BI6/J mice. Before and after Iso (30 mg/kg/day), we characterized LV morphology and function as well as global and segmental strain. We observed that Iso reduced LV ejection in both male $(-8.7 \%)$ and female $(-14.7 \%)$ mice. Several diastolic function parameters were negatively regulated in males and females such as E/A, E/E', isovolumetric relaxation time. Global longitudinal (GLS) and circumferential (GCS) strains were reduced by Iso in both sexes, GLS by $31 \%$ and GCS by about $20 \%$. For the segmental LV analysis, we measured strain, strain rate, reverse strain rate, peak speckle displacement and peak speckle velocity in the parasternal long axis. We observed that radial strain of the LV posterior segments were more severely modulated by Iso than those of the anterior wall in males. In females, on the other hand, both posterior and anterior wall segments were negatively impacted by Iso. Longitudinal strain showed similar results to the radial strain for both sexes. Strain rate, on the other hand, was only moderately changed by Iso. Reverse strain rate measurements (an index of diastolic function) showed that posterior LV segments were negatively regulated by Iso. We then studied the animals 5 and 17 weeks after Iso treatment. Compared to control mice, LV dilation was still present in males. Ejection fraction was decreased in mice of both sex compared to control animals. Diastolic function parameters, on the other hand, were back to normal. Taken together, our study indicates that segmental strain analysis can identify LV regions that are more negatively affected by a cardiotoxic agent such as Iso. In addition, cessation of Iso was not accompanied with a complete restoration of cardiac function after four months. 
1 Segmental analysis by speckle-tracking echocardiography of the left ventricle response to

2 Isoproterenol in male and female mice.

3

4 Walsh-Wilkinson Elisabeth,

5 Arsenault Marie,

6 Couet Jacques*

7

8 Groupe de recherche sur les valvulopathies, Centre de recherche de l'Institut universitaire de cardiologie

9 et de pneumologie de Québec, Université Laval, Quebec City, Quebec, Canada

10

11

12 * jacques.couet@med.ulaval.ca 


\section{Abstract}

14 We studied by conventional and speckle-tracking echocardiography, the response of the left ventricle (LV) to a three-week continuous infusion of Isoproterenol (Iso), a non-specific beta-adrenergic receptor agonist in male and female C57BI6/J mice. Before and after Iso ( $30 \mathrm{mg} / \mathrm{kg} /$ day), we characterized LV morphology and function as well as global and segmental strain. We observed that Iso reduced LV ejection in both male $(-8.7 \%)$ and female $(-14.7 \%)$ mice. Several diastolic function parameters were negatively regulated in males and females such as $E / A, E / E$, isovolumetric relaxation time. Global longitudinal (GLS) and circumferential (GCS) strains were reduced by Iso in both sexes, GLS by $31 \%$ and GCS by about $20 \%$. For the segmental LV analysis, we measured strain, strain rate, reverse strain rate, peak speckle displacement and peak speckle velocity in the parasternal long axis. We observed that radial strain of the LV posterior segments were more severely modulated by Iso than those of the anterior wall in males. In females, on the other hand, both posterior and anterior wall segments were negatively impacted by Iso. Longitudinal strain showed similar results to the radial strain for both sexes. Strain rate, on the other hand, was only moderately changed by Iso. Reverse strain rate measurements (an index of diastolic function) showed that posterior LV segments were negatively regulated by Iso. We then studied the animals 5 and 17 weeks after Iso treatment. Compared to control mice, LV dilation was still present in males. Ejection fraction was decreased in mice of both sex compared to control animals. Diastolic function parameters, on the other hand, were back to normal. Taken together, our study indicates that segmental strain analysis can identify LV regions that are more negatively affected by a cardiotoxic agent such as Iso. In addition, cessation of Iso was not accompanied with a complete restoration of cardiac function after four months. 
34

35

36

37

\section{Introduction}

Adrenergic overstimulation using Isoproterenol (Iso) is often used to induce cardiac toxicity in small rodent models [1-3]. Sometimes described as an HF model (systolic and/or diastolic), a cardiac hypertrophy model or a SIC model (Takotsubo-like syndrome) [4-9], Iso treatment of rats or mice has been the object of an important amount of literature.

Many regimens of Iso administration to rodents have been used in past studies such as a single high dose bolus, several injections over days as well as continuous infusion using osmotic micro-pumps implanted subcutaneously or intra-peritoneally, or micro-pellets at different dosages and for various duration $[2,8,10-12]$. This diversity of experimental set-ups makes the comparison between studies sometimes difficult, as the goals pursued by the authors were often different. In addition, as for many preclinical studies, most experiments were conducted in male animals and less often in females. In spontaneously hypertensive rats, it was observed that Iso treatment was more prone in induce LV dilation in males than in females [13]. In mice, more studies were performed using females but again, few studies studied in parallel both sexes [14].

Sex-related differences in the Iso model have been studied using echocardiography in C57BI6 mice infused for 7 or 14 days ( $10 \mathrm{mg} / \mathrm{kg} /$ day) but relatively few differences in either systolic or diastolic cardiac function were found. Most of the differences were identified at tissue level [14]. More recently, another study found no sex differences in mice treated with Iso for 14 days [15].

In the present study, we wished to document the left ventricular (LV) response to a beta-adrenergic receptor-mediated insult using the Iso mouse model and to identify possibly morphological and functional sex differences. We used a relatively high dosage of Iso (30 mg/kg/day) for a longer period (21 days). Using conventional and speckle tracking echocardiography (STE), we evaluated if LV dysfunction 
56 was present and then performed a regional study of this dysfunction. We also studied how male and

57 female mice echo parameters evolved 5 and 17 weeks post-Iso.

58 Our results indicate that Iso induced both systolic and diastolic function impairments in mice and that

59 only small sex differences were present in the extent of these. By STE, we showed that the response to

60 Iso is not homogeneously distributed throughout the LV walls as the posterior LV wall seems more

61 sensitive to Iso effects, at least in male mice. As for LV reverse remodeling taking place after Iso, dilation

62 in males was still present four months later. 


\section{Materials and Methods}

\section{Mouse model}

The present study was conducted within the Mouse Animal model of Sex Differences and Aging in heart Failure (MASDAF) study, which follows longitudinally C57B/6/J mice up to two years in order to investigate biological sex and aging effects on the LV response to an insult. In this sub-study, we compared the LV response of both males and females (age 8 weeks) from Jackson Laboratory (Bar Harbor, ME, USA). After a week of acclimatization, micro-osmotic pumps (cat. no.: 1004; Alzet, Cupertino, CA, USA), releasing Isoproterenol (Iso: 30 mg/kg/day; Sigma-Aldrich, Mississauga, Ont, Canada; $n=10$ mice/group) or vehicle (saline; Ctrl. $n=8$ mice/group) were implanted subcutaneously in the back of the neck and left for 21 days [16]. Experienced technicians for health and behavior monitored the animals daily during the protocol. The animals were weighed weekly. No mouse displayed markers associated with death or poor prognosis of quality of life, or specific signs of severe suffering or distress, which would have led to early and immediate euthanasia. Among those, significant loss or gain of weight, grooming and changes in behavior were monitored. The protocol was approved by the Université Laval's animal protection committee and followed the recommendations of the Canadian Council on Laboratory Animal Care (protocol \#2019-360, VRR-19-075).

\section{Echocardiography}

An echocardiography (Echo) exam was performed the day before Iso infusion started and three weeks later. Echo images were acquired on a Vevo 3100 imaging system (VisualSonics, FujiFilm, Toronto, Canada) by the same investigator and analyzed off-line using Vevo LAB software. The investigator was blinded for animal identification. Animals were anesthetized and placed in a supine position on a heated platform $\left(39^{\circ} \mathrm{C}\right)$. The concentration of isoflurane was maintained around $1.5-2.5 \%$, so that the heart rate was kept over 400 beats/minute. 
86

87

88

89

90

91

92

93

2D echo: M-mode images were recorded to measure diastolic LV walls thickness and chamber diameters from the parasternal long axis (PSLAX) view and the short axis (SAX) view at the papillary muscle level. LV mass was calculated by the VevoLab echo analysis software (VisualSonics) using the following equation: $1.053 \times\left[(E D D+P W+I V S W)^{3}-E D D^{3}\right] \times 0.8$. EDD: end-diastolic LV diameter, PW: posterior diastolic wall thickness and IVSW: interventricular septal diastolic wall thickness. Fractional shortening from M-mode images was calculated using the following equation: (EDD - ESD)/EDD where: ESD endsystolic LV diameter. Mitral flow was measured by pulsed wave Doppler from an apical four-chamber view. Early diastolic peak filling velocity (E wave), peak filling velocity at atrial contraction (A wave), E wave deceleration time and the E/A ratio were obtained. The early-diastolic peak velocity $\left(E^{\prime}\right)$, the latediastolic peak velocity $\left(A^{\prime}\right)$ of the mitral valve annulus and $E^{\prime} / A^{\prime}$ as well as $E / E^{\prime}$ were obtained using tissue Doppler. LV volumes, ejection fraction (EF), stroke volume and cardiac output were calculated using the Simpson's rule method from LV chamber area tracings.

Speckle-tracking echo: 2D echo B-mode loops were acquired from the LV PSLAX and analyzed using Vevo Strain software (VisualSonics). Images were acquired at the highest frame rate possible (232 frames/s) and strain analysis was performed in the radial and longitudinal axes as previously described [17]. Also calculated by the Vevo Strain software were the reverse strain rate (a diastolic function index), speckle displacement $(\mathrm{mm})$ and velocity $(\mathrm{mm} / \mathrm{s})$. Figure $1 \mathrm{~A}$ illustrates the various segments studied as well as the direction of strain or speckle displacement.

\section{Statistical analysis}

All data are expressed as mean \pm standard error of the mean (SEM). Statistical analyses were performed on the $\log _{10}$ of the data. Normality was assessed using the Shapiro-Wilk test. Intergroup comparisons were conducted using Student's T-test using GraphPad Prism 8.4, (GraphPad Software Inc., La Jolla, CA, 
108 USA). Data from tables 1 to 3 were analyzed using 2-way ANOVA and Holm-Sidak post-test. P<0.05 was 109 considered statistically significant. 


\section{Results}

111 Isoproterenol induces systolic and diastolic impairments in male and female mice.

112 Before implantation of the micro-osmotic pump, a complete echocardiography exam was performed for 113 each animal. Baseline values are listed in Table S1. With the exception of those related to the relative

114 size of the animals depending on their biological sex, systolic and diastolic function baseline parameters 115 were mostly similar between males and females.

116 Ten of these eighteen mice of each sex were then treated for three weeks with a continuous infusion of 117 Isoproterenol (Iso), a non-specific beta-adrenergic receptor agonist. On day 21, osmotic pumps were 118 removed and the day after, a second echo exam was performed and data were compared to controls 119 (Ctrl). As depicted in Table 1, Iso treatment had relatively similar effects in male and female mice on Mmode measurements. End systolic LV diameter (ESD) was increased in mice of both sexes resulting in a corresponding decrease in fractional shortening (-7.4\% for males and $-7.9 \%$ for females). SAX M-mode data were similar (not shown but included in the raw data). Both end-diastolic (EDV) and end-systolic volumes (ESV) were increased after Iso treatment, respectively by $23 \%$ and $49 \%$ in males and by $14 \%$

124 (not significant; ns) and 60\% in females. This resulted in lowered ejection fraction for both male (-8.7\%) 125 and female (-14.7\%) mice compared to control. Stroke volume was unchanged in males but tended to 126 decrease in females (ns). Cardiac output was lower in Iso-treated mice.

127 Iso treatment also influenced diastolic echo parameters (Table 2). The E wave measured by pulse-wave Doppler was decreased in male mice but not in females. We then measured $E^{\prime}$ and $A^{\prime}$ waves from the mitral valve annulus movements by tissue Doppler. The E' wave was significantly reduced by $34 \%$ in males, whereas the E/E' ratio was significantly increased by Iso (30\%). Only a tendency was registered 
133 In Table 3 is illustrated the evolution of global longitudinal (GLS) and global circumferential (GCS) strains

134 in male and female mice receiving Iso. Global strain measurements take into consideration the entire LV

135 wall comparing the relative changes in LV inner contour length during the cardiac cycle. GLS is calculated

136 from the PSLAX view and GCS from SAX. For both parameters, GLS and GCS, more negative values are

137 associated with better fractional change. In control animals, GLS and GCS values were similar between

138 the sexes. After 3 weeks of Iso infusion, GLS values became significantly less negative in both males

$139(+31 \%)$ and females (+31\%). As for GCS, it worsened similarly in males (+17\%) and females (+23\%) after

140 Iso.

141 Segmental analysis using speckle tracking echocardiography (STE) points toward a non-uniform LV

142 response to Iso in males

143 The LV was divided into six segments for the PSLAX view, as described in Figure 1A. This allowed us to

144 investigate if the effects of Iso infusion were distributed globally along the LV endocardial wall or if one

145 or many segments were more seriously affected than others were.

146 Using segmental analysis, we compared the strain at baseline (Pre-Iso) and after Iso in male and female

147 mice. We chose to use baseline values as control in order to limit the effects of intra-group variability

148 between animals. As illustrated in Figure 1B-E, radial and longitudinal strains were reduced by Iso in

149 males for all three posterior LV wall segments, whereas anterior segments strain was mostly unchanged.

150 Radial strains of two posterior wall segments (base and mid) was also reduced in females. In addition,

151 radial strain for all LV anterior wall segments was reduced. Average radial and longitudinal strains (all

152 segments; in black) were negatively modulated by Iso for both male and female mice.

153 We grouped data from the segmental strain analysis as from either the base, the mid-ventricle or the 154 apex regardless of the posterior or the anterior walls. We did the same for the anterior or the posterior

155 wall data that were analyzed together regardless if they originated from the base, mid-ventricle or apex

PeerJ reviewing PDF | (2020:11:55238:1:1:NEW 10 Feb 2021) 
156 [3]. As illustrated in Figure 1E-M, both radial and longitudinal strains were reduced for the base LV

157 segment in male and female mice. Mid LV radial strain was reduced for both sexes. This was also the

158 case for the longitudinal strain in males as well as the apex longitudinal strain in male and female

159 animals. We then compared if the posterior wall strain was more affected by Iso than the anterior wall.

160 In females, Iso decreased both radial and longitudinal strains to the same extent $(-30 \%)$. In males, the

161 radial strain was significantly decreased only for the posterior wall. The longitudinal strain was reduced

162 for both walls but this decrease was more important for the posterior wall than the anterior one (-39\%

163 vs. $-18 \%$, respectively).

164 We measured the indexed time-to-peak (T2P) for strain data. This represents the time for strain to reach

165 its maximal value from baseline (R-wave). We indexed the value for the duration of the cardiac cycle

$166(1 / \mathrm{HR})$ in order to take into account variations in heart rate between animals. Values are thus expressed

167 as a percentage of a cardiac cycle for strain to reach its peak. As illustrated in Figure S1, iT2P for each LV

168 segment in radial and longitudinal remained mostly stable in males. In females however, a greater

169 variation after Iso was observed suggesting that normal LV wall synchronicity was perturbed. We then

170 calculated the standard deviation (SD) of iT2P for each 6 segments. In males (blue), these parameters

171 remained stable after Iso but in females (orange), radial iT2P SD was increased.

172 As illustrated in Video S1 (males) and Video S2 (females), LV wall deformation after Iso was markedly

173 reduced. When LV movement tendency was expressed using velocity vectors as illustrated in Figure 2 , it

174 can be appreciated that in both systole and diastole, the reduction of the length and the changes in the

175 orientation of velocity vectors induced by Iso.

176 Peak strain rate (SR) represents a systolic function index that indicates the maximal rate of deformation

177 (strain) during systole. On the other hand, reverse peak SR happens during the passive LV filling phase of 178 diastole (Figure 3A) and has been proposed as a new index of diastolic function [18]. Changes in radial 
179

180

181

182

184

185

and longitudinal strain rates (SR) caused by Iso were relatively minor and concentrated on the posterior segments in males and females (Figure 3B-E). Peak reverse SR showed a pattern reminiscent of those of strain and SR. Iso negatively modified LV posterior segments in both males and females (Figure 3F-H). Several anterior wall segments were also affected, mostly in females.

Peak speckle displacement and peak speckle velocity are analogous to strain and strain rate although they are not expressed relative to a second point in the myocardium as for the strain and SR. Original speckle position or speckle velocity is determined at the R-wave and is arbitrarily fixed to zero. Iso reduced radial speckle displacement and velocity mainly for the posterior wall segments in males and females compared to baseline values. Displacement was also decreased for the anterior wall in females. In the longitudinal direction, peak speckle displacement remained stable in males and was reduced for only one segment (anterior base) in females. A similar situation was observed for longitudinal velocity of the LV wall (Figure S2).

After 21 days (week 3) of Iso treatment, the osmotic micro-pumps were removed and the LV reverse remodeling was studied by echo at week 8 and 20 or 5 and 17 weeks post-Iso, respectively. As illustrated in Figure 4A-J, LV end-systolic volume in males remained increased compared to control animals, whereas in females, no difference was present. Iso cessation helped reduce LV end-systolic volume in both males and females. However, only females returned to normal values at week 8 before increasing again 12 weeks later. This resulted that both male and female mice had a decreased ejection fraction four months post-Iso. On the other hand, both diastolic parameter, E/E' and IVRT, were normalized 5 weeks after Iso treatment. At the end of the follow-up, GLS and GCS had returned to normal for males but only GCS for females (Figure 4K-L). Segmental strain analysis showed a similar picture for males where strain values were unchanged compared to age-matched controls. On the other hand, both radial and longitudinal strains showed abnormalities in females 17 weeks after Iso treatment (Figure 4M). Posterior apical segment in radial and both apical segments showed diminished strain (Figure 4O-R). We 
203 also look at the reverse strain rate as a diastolic parameter. Compared to control animals, average

204 reverse SR in Iso-treated animals at week 20 were normal (Figure 4N). 
205

206

207

208

209

210

211

212

213

214

215

216

217

218

219

220

221

222

223

224

\section{Discussion}

In the present study, we demonstrated that the LV response to beta-adrenergic sur-activation using Iso, resulted in both systolic and diastolic function impairments in mice of both sexes. Using speckle-tracking echocardiography, we proceeded to a thorough investigation of the strain, strain rate and other related parameters. In males, we observed that the LV posterior wall was in general, more negatively affected by Iso than the anterior wall. This was true for the strain in both radial and longitudinal directions. In females, strain in the LV posterior wall was also negatively reduced but the anterior wall was also affected making the effects of Iso more global.

Most baseline values for the different STE parameters (strain, SR, and rSR) were similar between males and females. Radial peak speckle displacement and velocity for several segments were significantly smaller in females, which is likely related to a smaller heart size. To our knowledge, this study is the first to report segmental LV wall displacement and velocity data from normal young adult mice.

\section{Conventional echocardiography}

Systolic function as estimated by the ejection fraction (EF) was relatively more reduced in females than in males. Baseline EF values were higher in females, though. Various methods are available for the evaluation of EF by echo. The availability for small animals of four-dimensional (4D) echo LV chamber reconstruction over the cardiac cycle adds another way to estimate EF. In this study, we finally opted for the Simpson's method from LV chamber area tracings in PSLAX instead of 4D echo. Four-dimensional echo, in our hands, probably underestimated LV volume, which lead to incorrect calculations of LV volumes, stroke volume, ejection fraction and so forth. Depending on the method, EF estimates went from being in the $65-70 \%$ range using M-mode, $57-62 \%$ range using Simpson's method to $55-60 \%$ (PSLAX) and 45-50\% (SAX) using 4D echo in normal mice. In addition, Iso effects seemed to be masked using 4D echo and variability was high using this method. 
228 Several factors can limit acquisition of quality 4D echo scans in rodents as described before in several

229 studies $[15,19-21]$. One is interference from anatomical structures (sternum, ribs and lungs) that often

230 obscure parts of the heart, making it difficult to visualize and to trace LV walls. Working with those low-

231 quality 4D echo scans can significantly increase intra-observer variability and thus, reduce

232 reproducibility.

233 Despite these discrepancies between the methods used to evaluate EF, Iso increased both systolic LV

234 diameters and volumes in mice. A LV dilation was also apparent in 2D B-mode views, whereas the wall

235 thickness seemed to remain remained stable (see Figure 2, Videos S1 and S2). This LV remodeling helped

236 preserve cardiac output although a trend for a decrease was present. It is difficult to conclude that mice

237 were experiencing symptoms of HF, especially since cardiac output was mostly preserved. HF in small

238 rodents is often recognized by increased lungs weight after euthanasia or decreased exercise

239 performance [1]. We did not test resistance to forced exercise in our animals.

240 In the case of diastolic parameters measured by either pulse-wave or tissue Doppler, many of them

241 were negatively modulated by Iso in male mice. In females, only those measured by tissue Doppler were

242 affected by Iso and, to a lesser extent than for males. As in humans, defining diastolic dysfunction by

243 echo is difficult although clearly, Iso treatment caused diastolic function impairments, especially in male

244 mice.

245

246

247

248

249

250

Increased myocardial interstitial fibrosis is often related to diastolic dysfunction. Iso treatment has been shown to induce intertitial fibrosis in mice in other studies. Collagen production was shown to by more important in male mice receiving Iso than in females [14]. Among the other mechanisms that have been proposed to explain HF induced by Iso is an increased rate of cardiomyocyte apoptosis [22]. Suractivation of the beta-adrenergic system by Iso imposes an increased cardiac workload, which is associated with increased consumption of oxygen by the myocardium. This can lead to increased 
251 production of reactive oxygen species [23]. An additional mechanism for negative effects of Iso is

252 lipotoxicity. In an acute model of Iso-induced stress cardiomyopathy, rapid lipid accumulation was

253 noticed as soon as two hours after injection. It is not clear if here, in our chronic setting, that this

254 intracellular lipid accumulation is present or lasts for days but this does not exclude a possible toxicity of

255 Iso for the cardiac myocytes leading to apoptosis [8].

\section{Speckle-tracking echocardiography}

257 In this study, conventional echo highlighted LV anomalies both morphological and functional. Our study

258 design did not aim at testing if strain analysis was more sensitive for early detection of dysfunction as it

259 was done previously [10, 24-26]. Our goal was to evaluate if additional and valuable information could

260 be obtained using STE such as segmental strain and SR but also reverse SR, speckle displacement and

261 speckle velocity.

262 Using the strain analysis, we observed that the LV posterior wall was in general, more negatively

263 modulated by Iso than the anterior wall. The base and the mid-ventricle segments were the most

264 affected and the apex, to a lesser extent. This trend was clearer in males although it was also present in

265 females. Obviously, Iso treatment being longer and more severe in this study, triggered a compensatory

266 response [10]. LV dilatation had time to take place in males, which had to be accompanied by a

267 concomitant extracellular matrix remodeling. Fibrosis has been described in other studies looking at Iso

268 effects on the mouse myocardium $[15,22]$. This long exposure to Iso also likely brought a general down

269 regulation of beta-adrenergic receptors leaving non-receptor-mediated Iso effects play a significant part 270 here.

271 The reason for the posterior wall being more sensitive to Iso than the anterior one is not clear.

272 Differences in LV regional beta-receptor sub-types density have been reported before. Greater apical

273 beta-adrenergic receptor density or responsiveness has been described in humans, dogs, rats, cats and 
274 rabbit hearts [27-31]. Use of Iso infusion to create infarct-like damages and HF or for inducing

275 Takotsubo-like syndrome selectively targets the LV apex [8, 30-34]. This basal-apex gradient of beta-

276 adrenergic receptor responsiveness has thus been well-described. Here, our observation made in the

277 PSLAX now includes an additional axis for a possible antero-posterior gradient in beta-adrenergic

278 receptor sensitivity to sur-activation. Unfortunately, we could only describe this observation without

279 providing satisfactory explanations. The complex 3D architecture of the myocardium may provide clues,

280 but more studies are needed to provide new insights about this intriguing observation.

281 Speckle peak displacement data at baseline show that in the radial direction, posterior segments are

282 more mobile than anterior ones (Figure S2). This is associated with larger radial wall velocities for these

283 posterior LV segments. Here too, it was these posterior segments that were more negatively targeted by

284 Iso for both peak displacement and peak velocity. In the longitudinal direction, baseline values were

285 relatively similar to posterior and anterior segments.

286 As illustrated in Figure 4, velocity vectors originating from the LV base (and the apex) show a more

287 important longitudinal component than radial. For the mid-ventricle segments, it is the opposite and the

288 radial component of the velocity vectors is more important. Strain and SR measurements are expressed

289 relative to a baseline position at the initiation of systole (R-wave). They do not provide, however, a clear

290 evaluation of the changes in the direction (more radial or more longitudinal) of these vectors after Iso

291 treatment. These vectors also provide information about LV relaxation. This illustrates the high

292 complexity of cardiac contraction and relaxation and the difficulty to assess regional LV dynamic

293 response using only one dimension at a time, here radial vs. longitudinal.

294 Reverse strain rate has been proposed as an index of diastolic function in mice [18]. Considering that

295 most parameters measured by STE are systolic in nature, reverse strain rate can constitute an

296 interesting window to the kinetics of LV relaxation, at least during its passive filling. It is interesting to 
297 notice that again, the posterior wall segments in males are the ones with the more reduced reverse SR

298 suggesting LV stiffening caused by Iso seemed to mainly target this region.

299 STE strain analysis has been performed in the past in Iso-treated mice. In male mice receiving Iso for

300 either three or seven days, global radial and longitudinal strain and strain rate were reduced in PSLAX

301 and only strain rates were reduced in SAX. When concentrating on LV wall segments in PSLAX, the

302 authors found no regional differences suggesting that Iso effects on the LV after three or seven days

303 were mostly global [10]. Their dosage of Iso used was lower (5 vs. $30 \mathrm{mg} / \mathrm{kg}$ here) and duration of

304 treatment shorter, making it difficult to make comparison with our work. It is probable that in the

305 present study, more chronic mechanisms took place at the cellular and molecular levels, as mentioned

306 above. Interestingly, after only three days, An and collaborators observed in their mice, increased

307 myocardial fibrosis and hypertrophy. They did not mention if chamber dilatation was present but

308 reported LV wall thickening. We did not observe this in our mice after three weeks of Iso [10].

309 We thus observe a clear reduction of global strain measurements in our animals and this was similar

310 between males and females. These parameters are highly sensitive to detect cardiac dysfunction but do

311 not provide regional information. Since most sex differences we observed were present at the regional

312 level, global longitudinal and circumferential strains were not informative.

\section{A Takotsubo cardiomyopathy model?}

314 As mentioned above, Isoproterenol has been used to develop animal models of Takotsubo syndrome.

315 Takotsubo syndrome is a "transient LV dysfunction (hypokinesia, akinesia, or dyskinesia) presenting as

316 apical, midventricular, basal, or focal ballooning" [35]. The adrenergic overstimulation is believed to be

317 an important cause of this cardiomyopathy, which is more frequent in postmenopausal women

318 suggesting possible protective roles for estrogens and/or for the male sex [36].

Peer) reviewing PDF | (2020:11:55238:1:1:NEW 10 Feb 2021) 
319 The rat is usually the preferred animal model to study SIC. It has been studied acutely after a bolus

320 administration of Iso or after a few days of treatment. These short regimens usually allow complete

321 recuperation of systolic function in days or weeks following Iso $[5,7,12,37,38]$. Beta ${ }_{2}$-adrenergic

322 receptor sarcolemmal localization was proposed for being responsible for the typical apical ballooning

323 associated with Takotsubo cardiomyopathy [39]. Since mid-ventricular and basal forms of this SIC exist,

324 other mechanisms are involved.

325 One study had been conducted in mice using Iso (one single dose of $400 \mathrm{mg} / \mathrm{kg}$ ) to induce a Takotsubo-

326 like syndrome [8]. It did not result in global reduction of systolic function when assessed two hours after

327 a bolus Iso injection. Segmental fractional wall thickening was measured in SAX view in these mice.

328 Interestingly, two segments had their radial strain severely reduced namely the posterior wall and

329 inferior free wall segments. These segments in SAX are part of the posterior wall in PSLAX. The four

330 other LV segments had an increased strain to compensate in this study [8]. We did not observe this

331 pattern of compensation in our mice.

332 Most attempts to reproduce a Takotsubo-like syndrome in rodents have relied so far on acute

333 administration of Iso or catecholamines. This relies on the hypothesis that an acute surge of circulating

334 catecholamines levels or adrenergic overstimulation are important parts of SIC etiology in patients.

335 Known triggering factors in humans such as the death of a loved one, divorce, financial loss, diagnosis of

336 a serious disease all have an important chronic component. In addition, circulating levels of

337 catecholamines are seldom elevated in SIC patients [8]. This can make our study relevant to mimic

338 human SIC since mice received for a long period of time a beta-receptor agonist. One limitation is that

339 Iso does not stimulate alpha receptors as catecholamines do, eliminating the vasospasm component of

340 Takotsumo cardiomyopathy [8]. 
341 Interestingly, we showed that cessation of Iso was accompanied with improvement of systolic and

342 diastolic function parameters. Diastolic parameters quickly returned to normal 5 weeks post-Iso. It was

343 not the case for the systolic function. In females, the apex region strain got worse with time after initial

344 normalization 5 weeks post-Iso. This suggests that myocardial abnormalities remain long after Iso

345 cessation and that may lead to an eventual degradation of heart function. The reversible nature of

346 Takotsubo syndrome in patients has been questioned for some time in the literature [40]. If regional LV

347 contraction and global ejection fraction recover completely, other parameters of cardiac function can

348 remain abnormal including LV global longitudinal strain and LV diastolic function [41].

\section{Study limitations}

350 Among the limitations of this study, tissue data cannot be obtained since the animals were not

351 euthanized at the end of Iso treatment as their longitudinal follow-up was continued for several months.

352 Any tissue data in this context would have been from LVs having a long period of recuperation after Iso

353 treatment. It will be interesting to evaluate how sex hormones and age can influence LV response to

354 chronic Iso in the future. In addition, the study in short axis (SAX) at the level of the apex, the midsection

355 and the base the strain and related parameters could be informative to correlate changes observed here

356 in PSLAX.

\section{Conclusion}

Segmental strain analysis in mice can provide information about the regional influence of a toxic cardiac insult such as Iso continuous infusion. We observed both similarities and sex-related differences in our male and female mice. Systolic and diastolic functions were negatively modulated by Iso. Differences between sexes were relatively subtle when studied by conventional echo. LV dilation was more important in males and remained present post-Iso. By STE, we observed Iso had a more global effect on 
363 the female LV whereas in males, the posterior wall was more specifically targeted. Normalization after

364 Iso treatment was not complete for either male or female mice.

365

366

Acknowledgments

367

The authors want to acknowledge the technical help of Ms. Marine Clisson and Mr. Thomas Couët.

368

369

References

370

1. Gomes AC, Falcao-Pires I, Pires AL, Bràs-Silva C, Leite-Moreira AF. Rodent models of heart failure: an

371 updated review. Heart Fail Rev. 2013; 18: 219-49.doi:10.1007/s10741-012-9305-3.

372

2. Chang SC, Ren S, Rau CD, Wang JJ. Isoproterenol-Induced Heart Failure Mouse Model Using Osmotic Pump Implantation. Methods Mol Biol. 2018; 1816: 207-220. doi:10.1007/978-1-4939-8597-5-16.

3. Kudej RK, Iwase M, Uechi M, Vatner DE, Oka N, Ishikawa Y, Shannon RP, Bishop SP, Vatner SF. Effects of chronic beta-adrenergic receptor stimulation in mice. J Mol Cell Cardiol. 1997; 29: 2735-46.

376 doi:10.1006/jmcc.1997.0508.

4. Puhl SL, Weeks KL, Ranieri A, Avkiran M. Assessing structural and functional responses of murine hearts to acute and sustained -adrenergic stimulation in vivo. J Pharmacol Toxicol Methods. 2016; 79: 60-71. doi:10.1016/j.vascn.2016.01.007.

5. Wallner M, Duran JM, Mohsin S, Troupes CD, Vanhoutte D, Borghetti G, Vagnozzi RJ, Gross P, Yu D, Trappanese DM, Kubo H, Toib A, Sharp TE 3rd, Harper SC, Volkert MA, Starosta T, Feldsott EA, Berretta Myocyte Injury Without Cardiac Regeneration. Circ Res. 2016; 119: 865-79. 
384

385

386

387

388

389

390

391

392

393

394

395

396

397

398

399

400

401

402

403

404

doi:10.1161/circresaha.116.308687.

6. Sachdeva J, Dai W, Kloner RA. Functional and histological assessment of an experimental model of Takotsubo's cardiomyopathy. J Am Heart Assoc. 2014; 3: e000921. doi:10.1161/jaha.114.000921.

7. Shao Y, Redfors B, Scharin Täng M, Möllmann H, Troidl C, Szardien S, Hamm C, Nef H, Borén J,

Omerovic E. Novel rat model reveals important roles of -adrenoreceptors in stress-induced cardiomyopathy. Int J Cardiol. 2013; 168: 1943-50.

doi:10.1016/j.ijcard.2012.12.092.

8. Shao Y, Redfors B, Stahlman M, Täng MS, Miljanovic A, Möllmann H, Troidl C, Szardien S, Hamm C, Nef H, Borén J, Omerovic E. A mouse model reveals an important role for catecholamine-induced lipotoxicity in the pathogenesis of stress-induced cardiomyopathy. Eur J Heart Fail. 2013; 15: 9-22. doi:10.1093/eurjhf/hfs161.

9. Angelini P, Gamero MT. What can we learn from animal models of Takotsubo syndrome? Int J Cardiol. 2019; 281: 105-106. doi:10.1016/j.ijcard.2019.01.064.

10. An X, Wang J, Li H, Lu Z, Bai Y, Xiao H, Zhang Y, Song Y. Speckle Tracking Based Strain Analysis Is Sensitive for Early Detection of Pathological Cardiac Hypertrophy. PLoS One. 2016; 11: e0149155. doi:10.1371/journal.pone.0149155.

11. Ali A, Redfors B, Lundgren J, Alkhoury J, Oras J, Gan LM, Omerovic E. Effects of pretreatment with cardiostimulants and beta-blockers on isoprenaline-induced takotsubo-like cardiac dysfunction in rats. Int J Cardiol. 2019; 281: 99-104. doi:10.1016/j.ijcard.2018.12.045.

12. Ma X, Song Y, Chen C, Fu Y, Shen Q, Li Z, Zhang Y. Distinct actions of intermittent and sustained $\beta$ adrenoceptor stimulation on cardiac remodeling. Science China Life Sciences. 2011; 54: 493-501. 
405

406

407

408

409

410

411

412

413

414

415

416

417

418

419

420

421

422

423

424

13. Michel FS, Magubane M, Mokotedi L, Norton GR, Woodiwiss AJ. Sex-Specific Effects of AdrenergicInduced Left Ventricular Remodeling in Spontaneously Hypertensive Rats. J Card Fail. 2017; 23: 161-168. doi: 10.1016/j.cardfail.2016.09.017.

14. Zhu B, Liu K, Yang C, Qiao Y, Li Z. Gender-related differences in $\beta$-adrenergic receptor-mediated cardiac remodeling. Canadian journal of physiology and pharmacology. 2016; 94: 1349-1355.

15. Grant MKO, Abdelgawad IY, Lewis CA, Seelig D, Zordoky BN. Lack of sexual dimorphism in a mouse model of isoproterenol-induced cardiac dysfunction. PLoS One. 2020; 15: e0232507. doi:

10.1371/journal.pone.0232507.

16. Roussel E, Drolet MC, Lavigne AM, Arsenault M, Couet J. Multiple short-chain dehydrogenases/reductases are regulated in pathological cardiac hypertrophy. FEBS open bio. 2018; 8: 1624-1635.

17. Walsh-Wilkinson E, Drolet MC, Arsenault M, Couet J. Sex differences in the evolution of left ventricle remodeling in rats with severe volume overload. BMC Cardiovasc Disord 20, 51 (2020). https://doi.org/10.1186/s12872-020-01360-0.

18. Schnelle M, Catibog N, Zhang M, Nabeebaccus AA, Anderson G, Richards DA, Sawyer G, Zhang X, Toischer K, Hasenfuss G, Monaghan MJ, Shah AM. Echocardiographic evaluation of diastolic function in mouse models of heart disease. J Mol Cell Cardiol. 2018; 114: 20-28. doi:10.1016/j.yjmcc.2017.10.006.

19. Grune J, Blumrich A, Brix S, Jeuthe S, Drescher C, Grune T, Foryst-Ludwig A, Messroghli D, Kuebler WM, Ott C, Kintscher U. Evaluation of a commercial multi-dimensional echocardiography technique for ventricular volumetry in small animals. Cardiovascular ultrasound. 2018; 16: 10. 
425

426 427 2017; 3: 180.

20. Damen FW, Berman AG, Soepriatna AH, Ellis JM, Buttars SD, Aasa KL, Goergen CJ. High-frequency 4dimensional ultrasound (4DUS): a reliable method for assessing murine cardiac function. Tomography.

21. Rutledge C, Cater G, McMahon B, Guo L, Nouraie SM, Wu Y, Villanueva F, Kaufman BA. Commercial

4-dimensional echocardiography for murine heart volumetric evaluation after myocardial infarction.

Cardiovascular ultrasound. 2020; 18: 1-10.

22. Zhuo XZ, Wu Y, Ni YJ, Liu JH, Gong M, Wang XH, Wei F, Wang TZ, Yuan Z, Ma AQ, Song P.

Isoproterenol instigates cardiomyocyte apoptosis and heart failure via AMPK inactivation-mediated endoplasmic reticulum stress. Apoptosis. 2013; 18: 800-810. exercise training is AMPK-dependent. PloS one. 2015; 10: e0129971.

24. Li RJ, Yang J, Yang Y, Ma N, Jiang B, Sun QW, Li YJ. Speckle tracking echocardiography in the diagnosis of early left ventricular systolic dysfunction in type II diabetic mice. BMC Cardiovasc Disord. 2014; 14: 141. doi:10.1186/1471-2261-14-141.

25. Peng Y, Popovic ZB, Sopko N, Drinko J, Zhang Z, Thomas JD, Penn MS. Speckle tracking echocardiography in the assessment of mouse models of cardiac dysfunction. Am J Physiol Heart Circ Physiol. 2009; 297:H811-20. doi:10.1152/ajpheart.00385.2009.

26. Szymczyk E, Lipiec P, Plewka M, Białas M, Olszewska M, Rozwadowska N, Kamiński K, Kurpisz M, tracking in murine model of myocardial infarction: comparison with tissue Doppler echocardiography. J

445 Cardiovasc Med (Hagerstown). 2013; 14: 136-43. 
447 27. Mori H, Ishikawa S, Kojima S, Hayashi J, Watanabe Y, Hoffman JI, Okino H. Increased responsiveness

448 of left ventricular apical myocardium to adrenergic stimuli. Cardiovascular research. 1993; 27:192-198.

449 28. Kawano H, Okada R, Yano K. Histological study on the distribution of autonomic nerves in the human 450 heart. Heart and vessels. 2003; 18: 32-39.

451 29. Heather L, Catchpole A, Stuckey D, Cole M, Carr C, Clarke K. Isoproterenol induces in vivo functional 452 and metabolic abnormalities; similar to those found in the infarcted rat heart. Acta physiologica 453 Polonica. 2009; 12: 31.

454 30. Lathers CM, Levin RM, Spivey WH. Regional distribution of myocardial $\beta$-adrenoceptors in the cat. 455 European journal of pharmacology. 1986; 130: 111-117.

31. Mantravadi R, Gabris B, Liu T, Choi BR, de Groat WC, Ng GA, Salama G. Autonomic nerve stimulation reverses ventricular repolarization sequence in rabbit hearts. Circulation research. 2007; 100: e72-e80.

32. Paur H, Wright PT, Sikkel MB, Tranter MH, Mansfield C, O'Gara P, Stuckey DJ, Nikolaev VO, Diakonov Circulating Epinephrine Trigger Apical Cardiodepression in a $\beta_{2}$-Adrenergic Receptor/ $G_{i}$ Dependent Manner: A New Model of Takotsubo Cardiomyopathy. Circulation. 2012; 126:697-706. in the rat. Arch Pathol. 1959; 67: 443-455. regional perturbations of cardiac function in mouse models of cardiovascular disease. Echocardiography. 2013; 30:843-9. doi:10.1111/echo.12138. 
469 36. Sachdev E, Merz CNB, Mehta PK. Takotsubo cardiomyopathy. European Cardiology Review. 2015; 10:

47025.

471 37. Redfors B, Shao Y, Wikström J, Lyon AR, Oldfors A, Gan LM, Omerovic E. Contrast echocardiography

472 reveals apparently normal coronary perfusion in a rat model of stress-induced (Takotsubo)

473 cardiomyopathy. Eur Heart J Cardiovasc Imaging. 2014; 15: 152-7. doi:10.1093/ehjci/jet079.

474 38. Willis BC, Salazar-Cantú A, Silva-Platas C, Fernández-Sada E, Villegas CA, Rios-Argaiz E, González-

475 Serrano P, Sánchez LA, Guerrero-Beltrán CE, García N, Torre-Amione G, García-Rivas GJ, Altamirano J.

476 Impaired oxidative metabolism and calcium mishandling underlie cardiac dysfunction in a rat model of

477 post-acute isoproterenol-induced cardiomyopathy. Am J Physiol Heart Circ Physiol. 2015; 308:H467-77.

478 doi:10.1152/ajpheart.00734.2013.

479 39. Wright PT, Bhogal NK, Diakonov I, Pannell LMK, Perera RK, Bork NI, Schobesberger S, Lucarelli C, 480 Faggian G, Alvarez-Laviada A, Zaccolo M, Kamp TJ, Balijepalli RC, Lyon AR, Harding SE, Nikolaev VO, 481 Gorelik J. Cardiomyocyte Membrane Structure and cAMP Compartmentation Produce Anatomical

482 Variation in beta(2)AR-cAMP Responsiveness in Murine Hearts. Cell Rep. 2018; 23:459-469.

483 doi:10.1016/j.celrep.2018.03.053.

484 40. Pelliccia F, Kaski JC, Crea F, Camici PG. Pathophysiology of Takotsubo syndrome. Circulation. 2017;

$485 \quad 135: 2426-2441$.

486

487

488

489

490

41. Dias A, Núñez Gil IJ, Santoro F, Madias JE, Pelliccia F, Brunetti ND, Salmoirago-Blotcher E, Sharkey

SW, Eitel I, Akashi YJ, El-Battrawy I, Franco E, Akin I, Jaguszewski M, Dawson D, Figueredo VM, Napp LC, Christensen TE, Hebert K, Ben-Dor I, Ozaki Y, García-Garcia HM, Kajita AH, Akasaka T, Kurisu S, Lerman A, Waksman R. Takotsubo syndrome: State-of-the-art review by an expert panel - Part 2. Cardiovasc Revasc Med. 2019; 20: 153-166. doi: 10.1016/j.carrev.2018.11.016. 
491

492

493

494

495

496

497

498

499

500

501

502

503

504

505

506

507

508

509

510

511

512

513

\section{Legends to Figures}

Figure 1. Speckle tracking strain analysis. Radial and longitudinal strains can be obtained using the parasternal long axis (PSLAX) view. The six segments are also identified. Characters colors correspond to those used in the graphs for each of these LV segments. Ant: anterior, Post: posterior. Before after effects of Iso on the strain. Radial (A-B) and longitudinal (C-D) peak strains were obtained using the parasternal long axis view. The six segments are identified. Characters colors correspond to those used in the graphs for each of these LV segments. Radial (E-F and G-H) and longitudinal (I-J and K-L) peak strains were grouped either as from the base, the midsection or the apex or as from the anterior or the posterior segments in males (left panels) and females (right panels). Ant: anterior, Post: posterior. Males are represented on the left panels and females on the right. Results are represented as violin plots ( $n=8$ 10). Inner black lines represent quartiles of the data. Significance between groups was calculated with paired Student's T-test. *: $p<0.05,{ }^{* *}: p<\$ 0.01,{ }^{* * *}: p<\$ 0.001$ and $* * * *: p<0.0001$ between corresponding pre-Iso and Iso animals.

Figure 2. PSLAX LV wall trace tendency before and after Iso. LV wall trace tendency is expressed as velocity vectors for $>48$ points around the endocardium in systole $(A, C, E, G)$ and diastole $(B, D, F, H)$ before and after Iso in males (A-D) and female mice (E-H). Images of velocity vectors corresponds to the maximal peak (systole) and minimal peak (diastole) of the average curve of all six segments curves for speckle velocity in PSLAX. As evidenced by these PSLAX B-mode views, velocity vector orientation and length varies during the cardiac cycle. Vertical (radial) and horizontal (longitudinal) components of each vector do not correspond necessarily to the respective peak value of each orthogonal component.

Figure 3. A. Peak SR and Peak reverse SR (rSR). In the background is represented a screen caption of an M-mode loop of three cardiac cycles. In green, the EKG is superposed at the top. Radial strain rate curves are depicted for each SAX segment and an "average" curve in black. Notice that all curves begin 
514 at the R wave of the EKG. As evidenced by this representation, the first SR peak (top yellow circle)

515 corresponds to the maximal SR (1/s) whereas the second peak (bottom yellow circle) is inverted and

516 happens during the early stage of LV relaxation as evidenced by the M-mode image underneath. A male

517 mouse after three weeks of Iso is represented. B-H. Before after effects of Iso on strain rate (SR) and

518 reverse strain rate (rSR). Radial (B-C) and longitudinal (D-E) peak SR and rSR (F-H) are illustrated.

519 Characters colors correspond to those used in the graphs for each of these LV segments. Ant: anterior,

520 Post: posterior, SW: septal wall and FW: free wall. Males are represented on the left panels and females

521 on the right. Results are represented as violin plots $(n=8-10)$. Inner black lines represent quartiles of the

522 data. Significance between groups was calculated with paired Student's T-test. *: $p<0.05,{ }^{* *}: p<0.01$ and

$523 * * *: p<0.001$ between corresponding pre-Iso and Iso groups.

524 Figure 4. Reversibility of Iso treatment. A-J: Head-to-head longitudinal comparison of several echo 525 parameters of control (open circles) and Iso-treated (closed circles) male (blue) and female (orange)

526 mice. EDV: end-diastolic volume, ESV: end-systolic volume, EF: ejection fraction and IVRT: Isovolumetric

527 relaxation time. ${ }^{*}: p<0.05,{ }^{* *}: p<\$ 0.01,{ }^{* * *}: p<\$ 0.001$ and $* * * *: p<0.0001$ between corresponding

528 control and Iso animals using unpaired Student's T-test. \#: $p<0.05, \# \#$ : $p<0.01, \# \#$ : $p<0.001$ and \#\#\#:

$529 p<0.0001$ between measurements at different times using ANOVA and Tukey's post-test. K-L: Global

530 longitudinal strain (GLS) and global circumferential strain (GCS) at week 20 in male and female mice

531 treated or not with Iso for 3 weeks. M-N: Average radial and longitudinal peak strains at week 20. O-R:

532 Radial and longitudinal peak strains at week 20 in male and female mice treated or not with Iso. The six

533 segments are identified. Results are represented as violin plots $(n=8-10)$. Inner black lines represent

534 quartiles of the data. Significance between groups was calculated with unpaired Student's T-test. *:

$535 p<0.05,{ }^{* *}: p<0.01$ and ${ }^{* * *}: p<0.001$ between corresponding control (Ctrl) and Iso animals. 


\section{Table $\mathbf{1}$ (on next page)}

Left ventricle morphology and systolic function in male and female mice receiving or not Isoproterenol for three weeks.

Ctrl: control, Iso: Isoproterenol treatment, BW: body weight, LV: left ventricle mass, EDD: end diastolic LV diameter, ESD: end-systolic diameter, IVS: interventricular septum, PW: posterior wall, RWT: relative wall thickness, FS: fractional shortening, EDV: end-diastolic volume, ESV: end-systolic volume, SV: stroke volume, EF: ejection fraction, HR: heart rate, bpm: beats per minute and CO: cardiac output. Values are expressed as the mean \pm SEM. Two-way ANOVA statistical analysis results are displayed for each factor, sex and Iso, respectively. Intergroup $p$ values were calculated using Holm-Sidak post-test. a: $p<0.05, b: p<0.01, c: p<0.001$ and d: $p<0.0001$ between Ctrl and Iso groups, respectively. ns: not significant. 
1

Table 1.

2

\begin{tabular}{|c|c|c|c|c|c|c|c|}
\hline Parameters & $\begin{array}{c}M \text { Ctrl, } N= \\
8\end{array}$ & $\begin{array}{c}\text { M Iso, } N= \\
10\end{array}$ & $\begin{array}{c}\mathrm{F} \text { Ctrl, } \mathrm{N}= \\
8\end{array}$ & $\begin{array}{c}\text { F Iso, } N= \\
10\end{array}$ & Sex & Iso & $\begin{array}{c}\text { Sex X } \\
\text { Iso }\end{array}$ \\
\hline BW, g & $25.0 \pm 0.31$ & $27.9 \pm 0.53 b$ & $19.9 \pm 0.28$ & $20.8 \pm 0.45$ & $<0.0001$ & ns & ns \\
\hline Tibia, mm & $21.0 \pm 0.08$ & $21.7 \pm 0.10 \mathrm{~d}$ & $20.5 \pm 0.08$ & $20.3 \pm 0.13$ & $<0.0001$ & ns & ns \\
\hline \multicolumn{8}{|l|}{ M-mode } \\
\hline $\mathrm{EDD}, \mathrm{mm}$ & $3.8 \pm 0.03$ & $3.9 \pm 0.07$ & $3.6 \pm 0.06$ & $3.6 \pm 0.07$ & $<0.0001$ & ns & ns \\
\hline $\mathrm{ESD}, \mathrm{mm}$ & $2.6 \pm 0.08$ & $2.9 \pm 0.11 \mathrm{a}$ & $2.3 \pm 0.05$ & $2.6 \pm 0.07 b$ & $<0.01$ & $<0.001$ & ns \\
\hline IVS, mm & $0.82 \pm 0.02$ & $0.82 \pm 0.02$ & $0.76 \pm 0.01$ & $0.77 \pm 0.02$ & $<0.001$ & ns & ns \\
\hline $\mathrm{PW}, \mathrm{mm}$ & $0.84 \pm 0.01$ & $0.83 \pm 0.02$ & $0.78 \pm 0.01$ & $0.75 \pm 0.02$ & $<0.0001$ & ns & ns \\
\hline RWT & $0.44 \pm 0.01$ & $0.42 \pm 0.01$ & $0.43 \pm 0.01$ & $0.42 \pm 0.01$ & $\mathrm{~ns}$ & $\mathrm{~ns}$ & ns \\
\hline $\mathrm{FS}, \%$ & $32.2 \pm 1.69$ & $24.8 \pm 1.52 b$ & $35.4 \pm 0.98$ & $27.5 \pm 0.86 c$ & $<0.05$ & $<0.0001$ & ns \\
\hline $\mathrm{LV}, \mathrm{mg}$ & $86 \pm 2.1$ & $95 \pm 4.1$ & $76 \pm 2.6$ & $75 \pm 2.7$ & $<0.0001$ & ns & ns \\
\hline $\mathrm{iLV}, \mathrm{mg} / \mathrm{g}$ & $3.4 \pm 0.10$ & $3.4 \pm 0.11$ & $3.8 \pm 0.14$ & $3.6 \pm 0.11$ & $<0.01$ & ns & $\mathrm{ns}$ \\
\hline \multicolumn{8}{|l|}{ Simpson's } \\
\hline EDV,$\mu l$ & $54.6 \pm 1.83$ & $66.9 \pm 3.62 b$ & $44.6 \pm 0.74$ & $51.0 \pm 2.45$ & $<0.0001$ & $<0.001$ & ns \\
\hline $\mathrm{ESV}, \mu \mathrm{l}$ & $23.4 \pm 0.72$ & $34.8 \pm 2.78 \mathrm{~b}$ & $17.1 \pm 0.34$ & $27.4 \pm 2.13 b$ & $<0.01$ & $<0.0001$ & ns \\
\hline $\mathrm{SV}, \mathrm{mm}$ & $31.2 \pm 1.39$ & $32.1 \pm 1.91$ & $27.5 \pm 0.56$ & $23.6 \pm 1.19$ & $<0.001$ & ns & ns \\
\hline$E F, \%$ & $57.1 \pm 1.01$ & $48.4 \pm 2.19 a$ & $61.7 \pm 0.55$ & $47.0 \pm 2.80 c$ & ns & $<0.0001$ & ns \\
\hline $\mathrm{HR}, \mathrm{bpm}$ & $494 \pm 17.9$ & $415 \pm 10.0 \mathrm{~b}$ & $441 \pm 16.8$ & $448 \pm 9.0$ & ns & $<0.05$ & $<0.01$ \\
\hline $\mathrm{CO}, \mathrm{ml} / \mathrm{min}$ & $15.3 \pm 0.69$ & $13.3 \pm 0.73$ & $12.2 \pm 0.56$ & $10.6 \pm 0.60$ & $<0.0001$ & $<0.01$ & ns \\
\hline
\end{tabular}

3

4

5 


\section{Table 2 (on next page)}

Left ventricle diastolic parameters in male and female mice receiving or not Isoproterenol for three weeks.

Dec: deceleration time, IVRT: Isovolumetric relaxation time. Values are expressed as the mean \pm SEM. Two-way ANOVA statistical analysis results are displayed for each factor, sex and Iso, respectively. Intergroup p values were calculated using Holm-Sidak post-test. a: $p<0.05, b: p<0.01, c: p<0.001$ and $d: p<0.0001$ between Ctrl and Iso groups, respectively. ns: not significant. 
1 Table 2.

\begin{tabular}{|l|c|c|c|c|c|c|c|}
\hline Parameters & $M$ Ctrl, $\mathrm{N}=8$ & $\mathrm{M}$ Iso, $\mathrm{N}=10$ & $\mathrm{~F}$ Ctrl, $\mathrm{N}=8$ & $\mathrm{~F}$ Iso, $\mathrm{N}=10$ & Sex & Iso & Sex $\mathrm{X}$ Iso \\
\hline $\mathrm{E}, \mathrm{mm} / \mathrm{s}$ & $662 \pm 23.6$ & $551 \pm 18,4 \mathrm{c}$ & $578 \pm 15.6$ & $579 \pm 14.2$ & $\mathrm{~ns}$ & $<0.01$ & $<0.01$ \\
\hline $\mathrm{A}, \mathrm{mm} / \mathrm{s}$ & $430 \pm 21.7$ & $380 \pm 9.0$ & $363 \pm 10.1$ & $375 \pm 16.9$ & $<0.05$ & $\mathrm{~ns}$ & $\mathrm{~ns}$ \\
\hline $\mathrm{E} / \mathrm{A}$ & $1.55 \pm 0.03$ & $1.45 \pm 0.03$ & $1.60 \pm 0.05$ & $1.57 \pm 0.07$ & $\mathrm{~ns}$ & $\mathrm{~ns}$ & $\mathrm{~ns}$ \\
\hline $\mathrm{E} \mathrm{dec}, \mathrm{ms}$ & $20.0 \pm 1.20$ & $25.2 \pm 1.65$ & $18.8 \pm 0.70$ & $22.4 \pm 1.88$ & $\mathrm{~ns}$ & $<0.01$ & $\mathrm{~ns}$ \\
\hline $\mathrm{E}^{\prime}, \mathrm{mm} / \mathrm{s}$ & $27.6 \pm 1.49$ & $18.3 \pm 1.04 \mathrm{~d}$ & $26.1 \pm 1.61$ & $22.3 \pm 0.81$ & $\mathrm{~ns}$ & $<0.0001$ & $\mathrm{~ns}$ \\
\hline $\mathrm{A}^{\prime}, \mathrm{mm} / \mathrm{s}$ & $19.3 \pm 0.44$ & $13.8 \pm 0.98 \mathrm{c}$ & $20.4 \pm 1.41$ & $16.4 \pm 0.49 \mathrm{a}$ & $\mathrm{ns}$ & $<0.0001$ & $\mathrm{~ns}$ \\
\hline $\mathrm{E}^{\prime} \mathrm{E}^{\prime}$ & $24.2 \pm 1.45$ & $31.4 \pm 1.57 \mathrm{a}$ & $22.3 \pm 1.42$ & $30.5 \pm 2.54$ & $<0.05$ & $<0.001$ & $\mathrm{~ns}$ \\
\hline $\mathrm{E}^{\prime} / \mathrm{A}^{\prime}$ & $1.43 \pm 0.06$ & $1.36 \pm 0.04$ & $1.29 \pm 0.04$ & $1.43 \pm 0.06$ & $\mathrm{~ns}$ & $\mathrm{~ns}$ & $\mathrm{~ns}$ \\
\hline IVRT, $\mathrm{ms}^{\prime}$ & $15.1 \pm 0.32$ & $21.1 \pm 0.57 \mathrm{~d}$ & $16.1 \pm 0.61$ & $19.4 \pm 0.93 \mathrm{~b}$ & $\mathrm{~ns}$ & $<0.0001$ & $<0.05$ \\
\hline
\end{tabular}

2 


\section{Table 3 (on next page)}

Global LV strain parameters in male and female mice treated or not with Isoproterenol for three weeks.

GLS: global longitudinal strain and GCS: global circumferential strain. Values are expressed as the mean \pm SEM. Two-way ANOVA statistical analysis results are displayed for each factor, sex and Iso, respectively. Intergroup p values were calculated using Holm-Sidak post-test. c: $p<0.001$ and $d: p<0.0001$ between Ctrl and Iso groups, respectively. ns: not significant. 
1 Table 3.

\begin{tabular}{|l|c|c|c|c|c|c|c|}
\hline Parameters & $\mathrm{M}$ Ctrl, $\mathrm{N}=8$ & $\mathrm{M}$ Iso, $\mathrm{N}=10$ & $\mathrm{~F}$ Ctrl, $\mathrm{N}=8$ & $\mathrm{~F}$ Iso, $\mathrm{N}=10$ & Sex & Iso & Sex X Iso \\
\hline GLS & $-19.1 \pm 0.6$ & $-13.1 \pm 0.8 \mathrm{~d}$ & $-20.3 \pm 0.8$ & $-14.0 \pm 0.9 \mathrm{c}$ & $\mathrm{ns}$ & $<0.0001$ & $\mathrm{~ns}$ \\
\hline GCS & $-27.6 \pm 1.5$ & $-18.3 \pm 1.0 \mathrm{~d}$ & $-26.1 \pm 1.6$ & $-22.2 \pm 0.8$ & $\mathrm{~ns}$ & $<0.0001$ & $<0.05$ \\
\hline
\end{tabular}

2 


\section{Figure 1}

Speckle tracking strain analysis.

Radial and longitudinal strains can be obtained using the parasternal long axis (PSLAX) view. The six segments are also identified. Characters colors correspond to those used in the graphs for each of these LV segments. Ant: anterior, Post: posterior. Before after effects of Iso on the strain. Radial (A-B) and longitudinal (C-D) peak strains were obtained using the parasternal long axis view. The six segments are identified. Characters colors correspond to those used in the graphs for each of these LV segments. Radial (E-F and G-H) and longitudinal (I-J and K-L) peak strains were grouped either as from the base, the midsection or the apex or as from the anterior or the posterior segments in males (left panels) and females (right panels). Ant: anterior, Post: posterior. Males are represented on the left panels and females on the right. Results are represented as violin plots $(n=8-10)$. Inner black lines represent quartiles of the data. Significance between groups was calculated with paired Student's T-test. *: $p<0.05, * *: p<\$ 0.01, * * *: p<\$ 0.001$ and ****: $p<0.0001$ between corresponding pre-Iso and Iso animals. 
B

\section{Males}

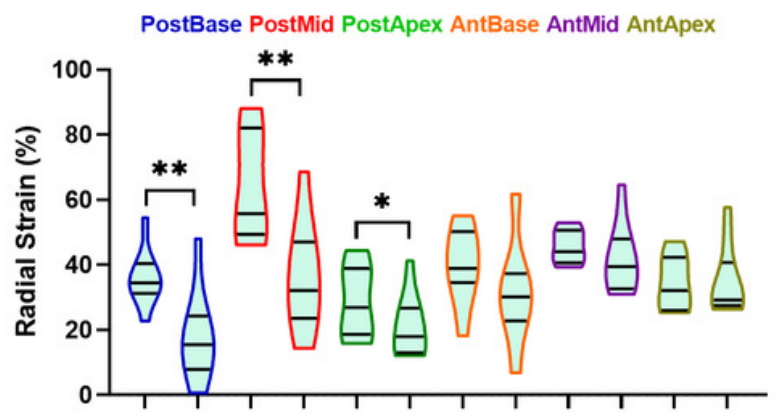

D

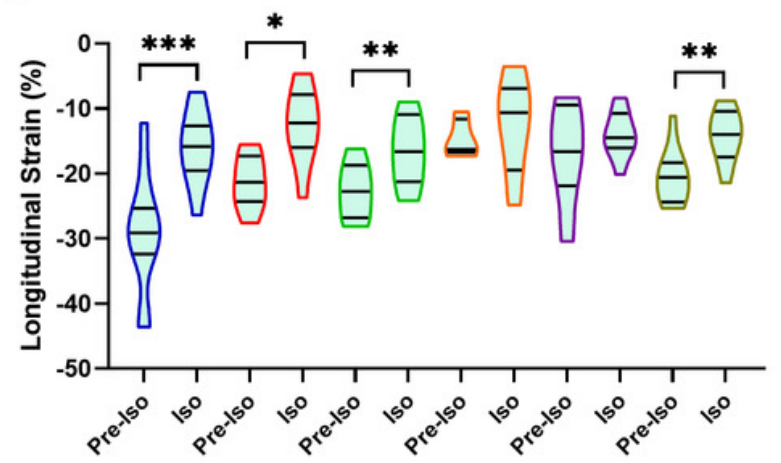

$\mathbf{F}$
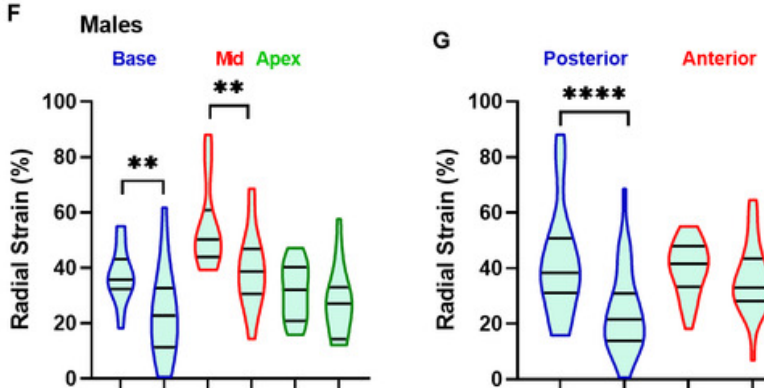

J

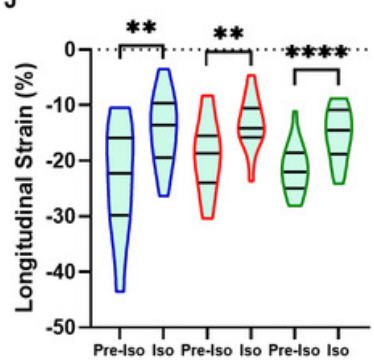

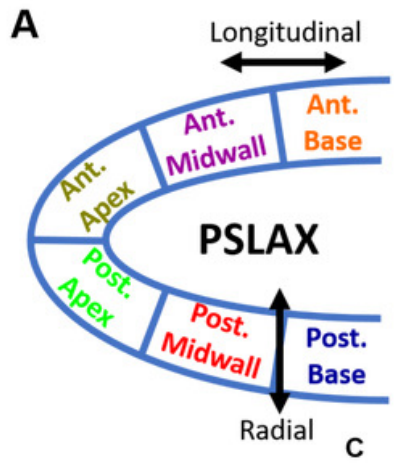

Females

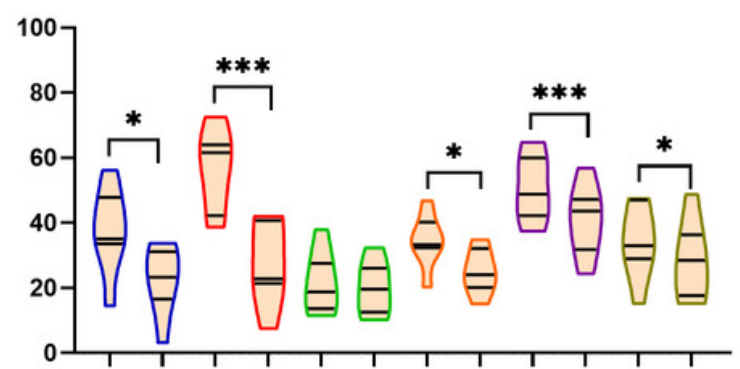

E

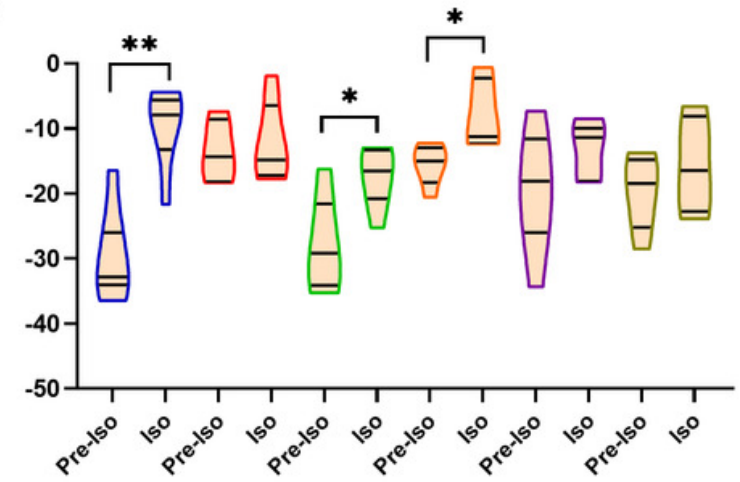

H

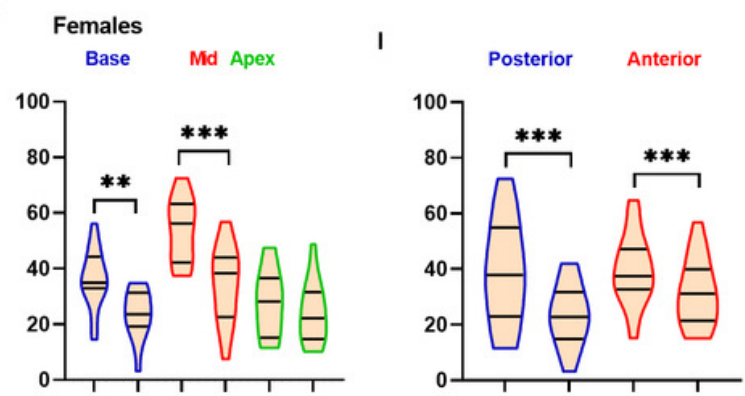

L

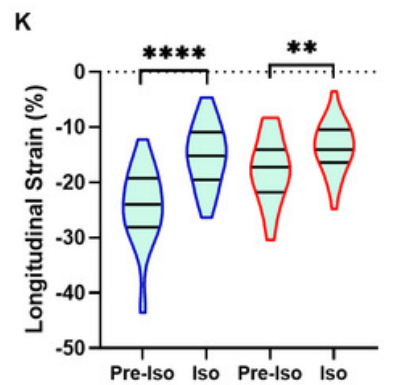

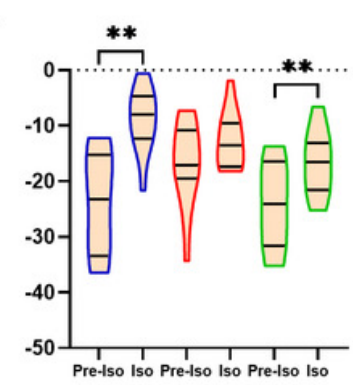

M

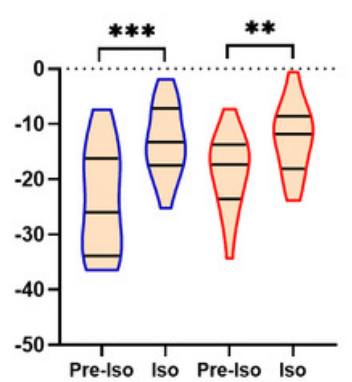




\section{Figure 2}

PSLAX LV wall trace tendency before and after Iso.

LV wall trace tendency is expressed as

velocity vectors for $>48$ points around the endocardium in systole (left panels) and diastole (right panels) before and after Iso in males (top panels) and female mice (bottom panels). Images of velocity vectors corresponds to the maximal peak (systole) and minimal peak (diastole) of the average curve of all six segments curves for speckle velocity in PSLAX. As evidenced by these PSLAX B-mode views, velocity vector orientation and length varies during the cardiac cycle. Vertical (radial) and horizontal (longitudinal) components of each vector do not correspond necessarily to the respective peak value of each orthogonal component. 

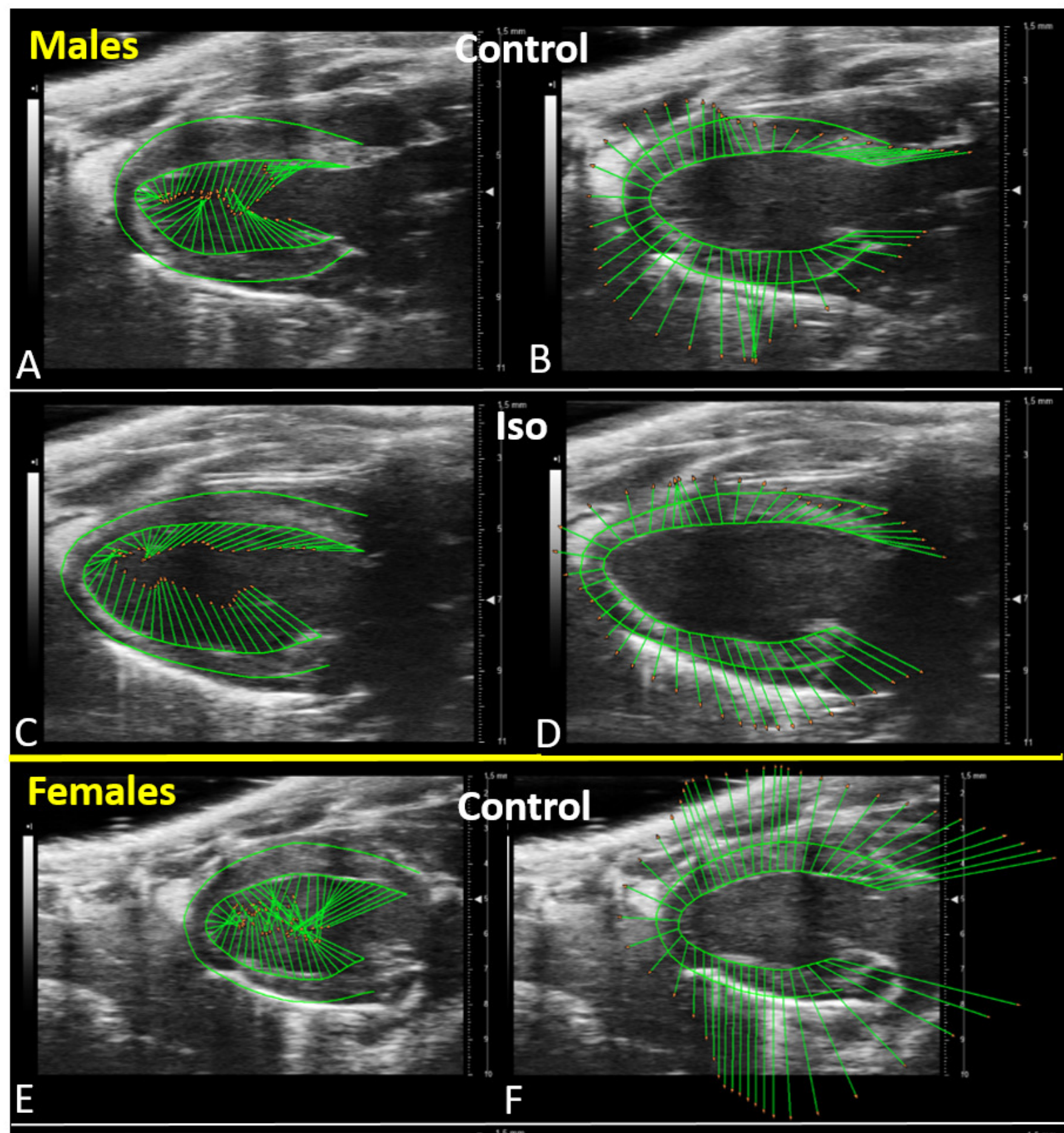

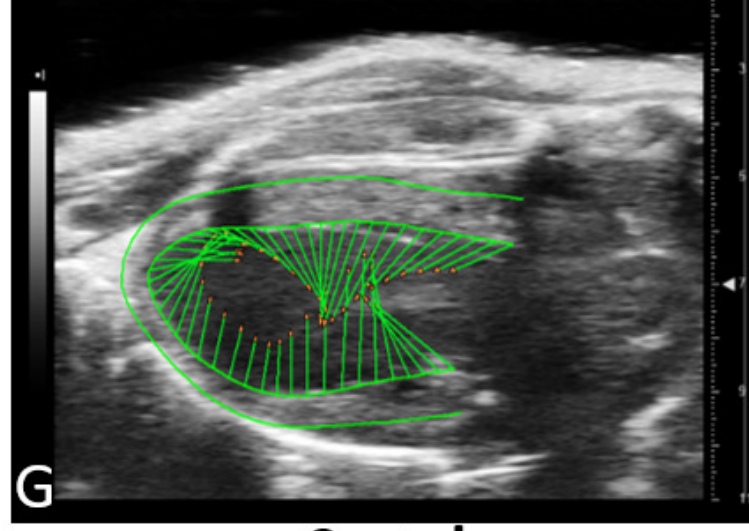

Systole

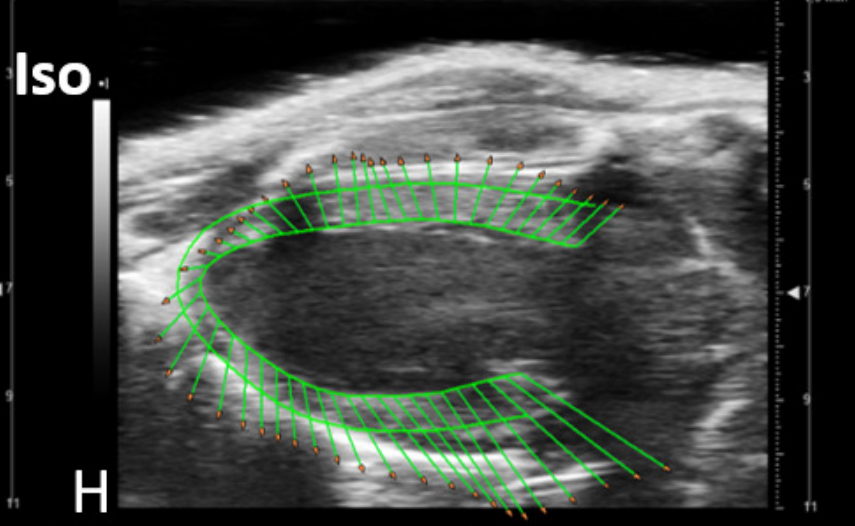

Diastole 


\section{Figure 3}

Peak SR and Peak reverse SR (rSR).

In the background is represented a screen caption of an M-mode loop of three cardiac cycles. In green, the EKG is superposed at the top. Radial strain rate curves are depicted for each SAX segment and an "average" curve in black. Notice that all curves begin at the $\mathrm{R}$ wave of the EKG. As evidenced by this representation, the first SR peak (top yellow circle) corresponds to the maximal SR (1/s) whereas the second peak (bottom yellow circle) is inverted and happens during the early stage of LV relaxation as evidenced by the M-mode image underneath. A male mouse after three weeks of Iso is represented. B-H. Before after effects of Iso on strain rate (SR) and reverse strain rate ( $\mathrm{rSR}$ ). Radial (B-C) and longitudinal (D-E) peak SR and rSR (F-H) are illustrated. Characters colors correspond to those used in the graphs for each of these LV segments. Ant: anterior, Post: posterior, SW: septal wall and FW: free wall. Males are represented on the left panels and females on the right. Results are represented as violin plots ( $n=8-10)$. Inner black lines represent quartiles of the data. Significance between groups was calculated with paired Student's T-test. *: $p<0.05, * *$ : $p<0.01$ and $* * *: p<0.001$ between corresponding pre-Iso and Iso groups. 
A

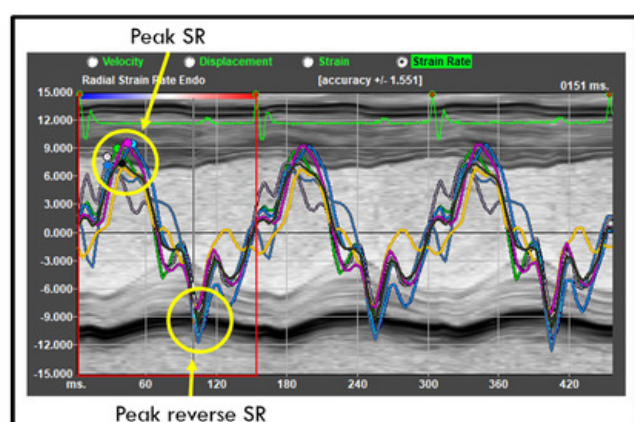

B

Males

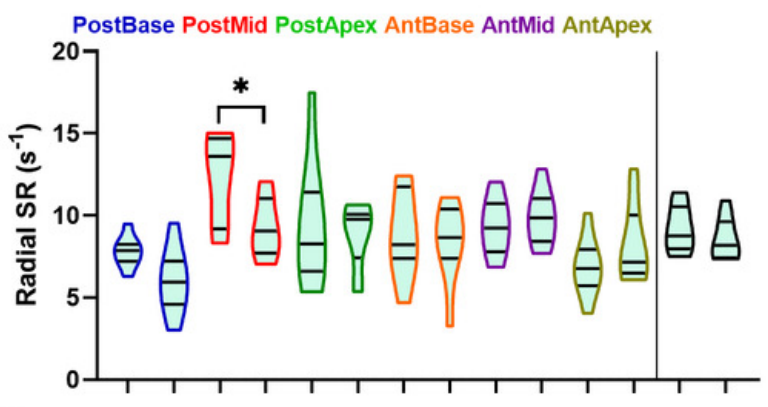

D

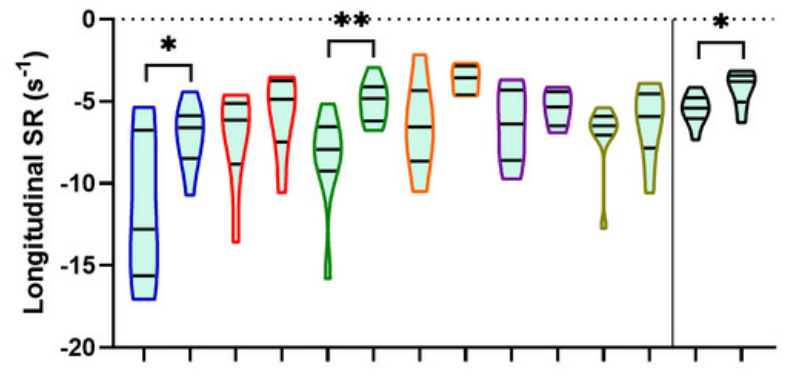

F

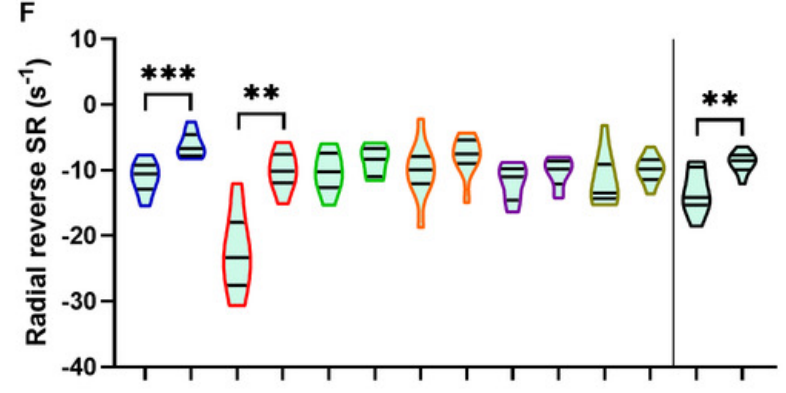

H

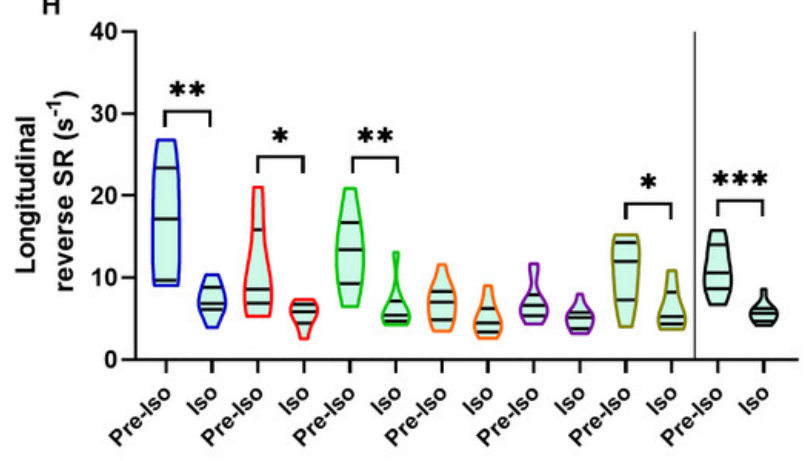

C Females

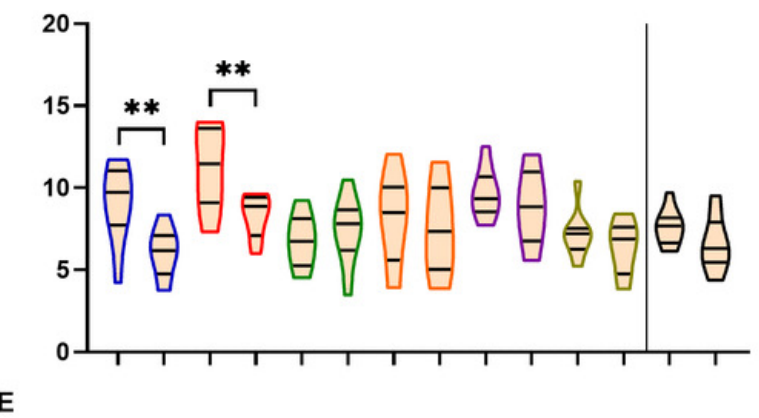

E

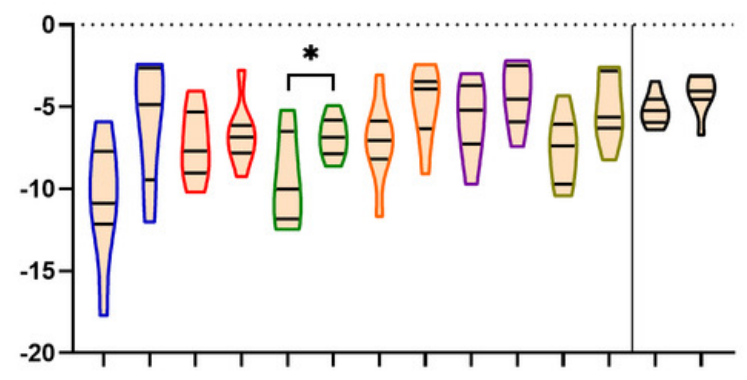

G
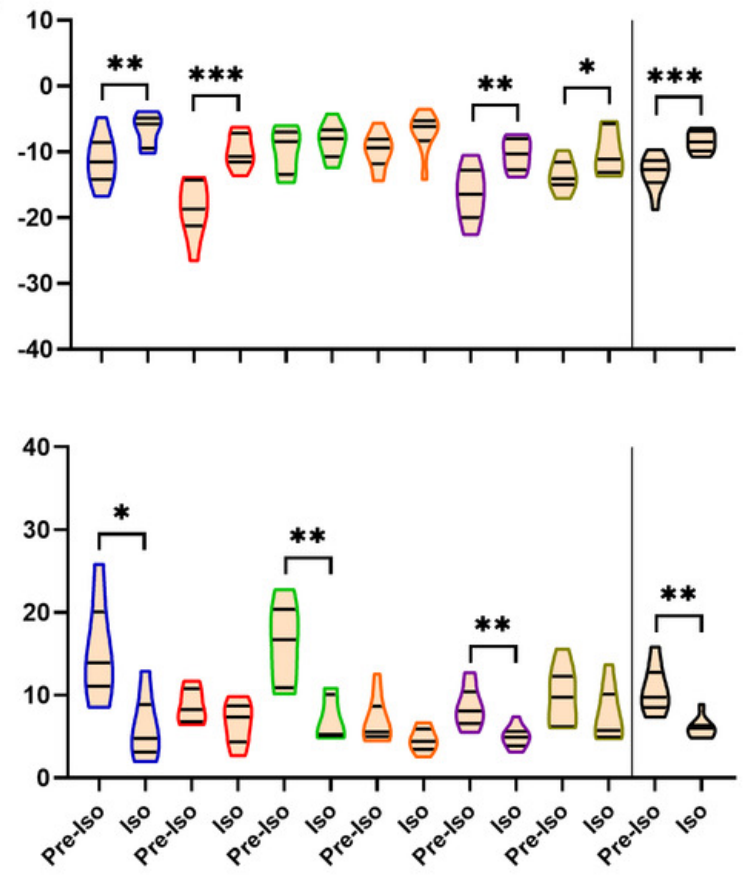


\section{Figure 4}

Reversibility of Iso treatment.

A-J: Head-to-head longitudinal comparison of several echo parameters of control (open circles) and Iso-treated (closed circles) male (blue) and female (orange) mice. EDV: enddiastolic volume, ESV: end-systolic volume, EF: ejection fraction and IVRT: Isovolumetric relaxation time. $*: p<0.05, * *: p<\$ 0.01, * * *: p<\$ 0.001$ and ****: $p<0.0001$ between corresponding control and Iso animals using unpaired Student's T-test. \#: $p<0.05$, \#\#: $p<0.01$, \#\#: $p<0.001$ and \#\#\#: $p<0.0001$ between measurements at different times using ANOVA and Tukey's post-test. K-L: Global longitudinal strain (GLS) and global circumferential strain (GCS) at week 20 in male and female mice treated or not with Iso for 3 weeks. M-N: Average radial and longitudinal peak strains at week 20. O-R: Radial and longitudinal peak strains at week 20 in male and female mice treated or not with Iso. The six segments are identified. Results are represented as violin plots $(n=8-10)$. Inner black lines represent quartiles of the data. Significance between groups was calculated with unpaired Student's T-test. *: $p<0.05, * *: p<0.01$ and $* * *: p<0.001$ between corresponding control (Ctrl) and Iso animals. 
A

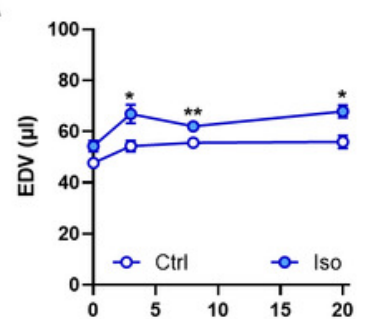

B

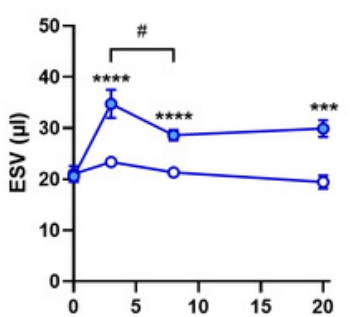

C
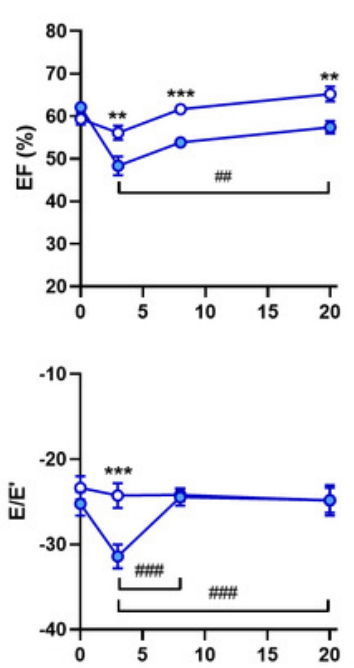

E

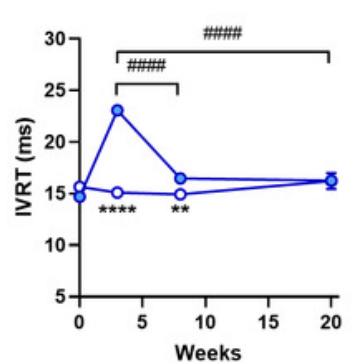

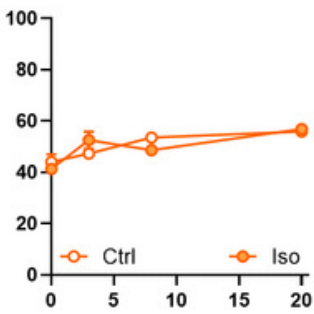

G

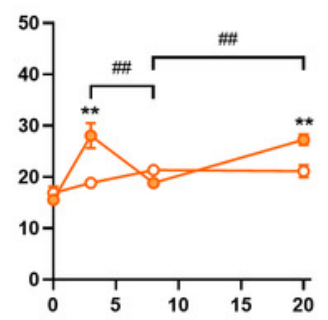

H
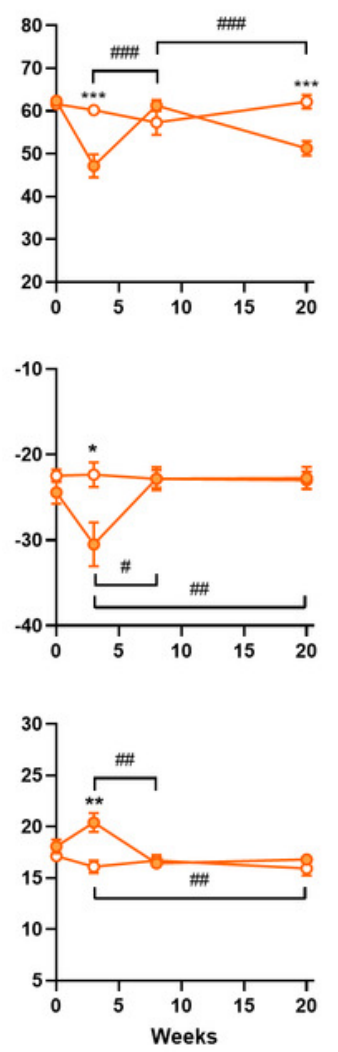

K
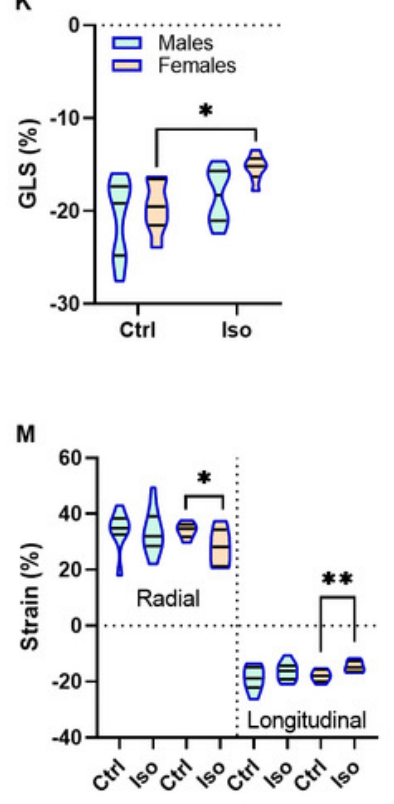
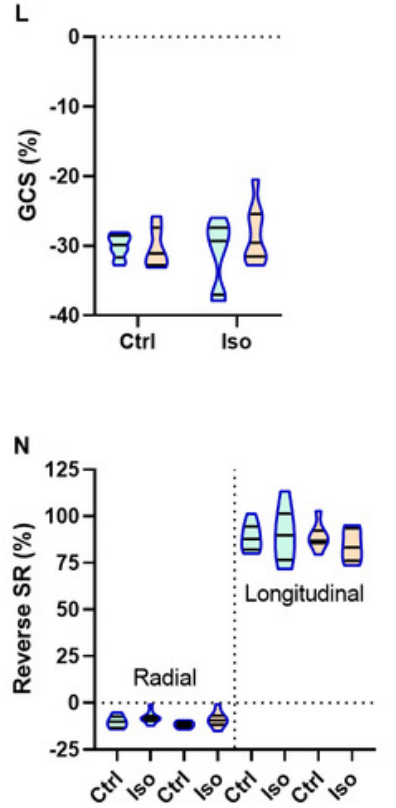

PostBase PostMid PostApex AntBase AntMid AntApex
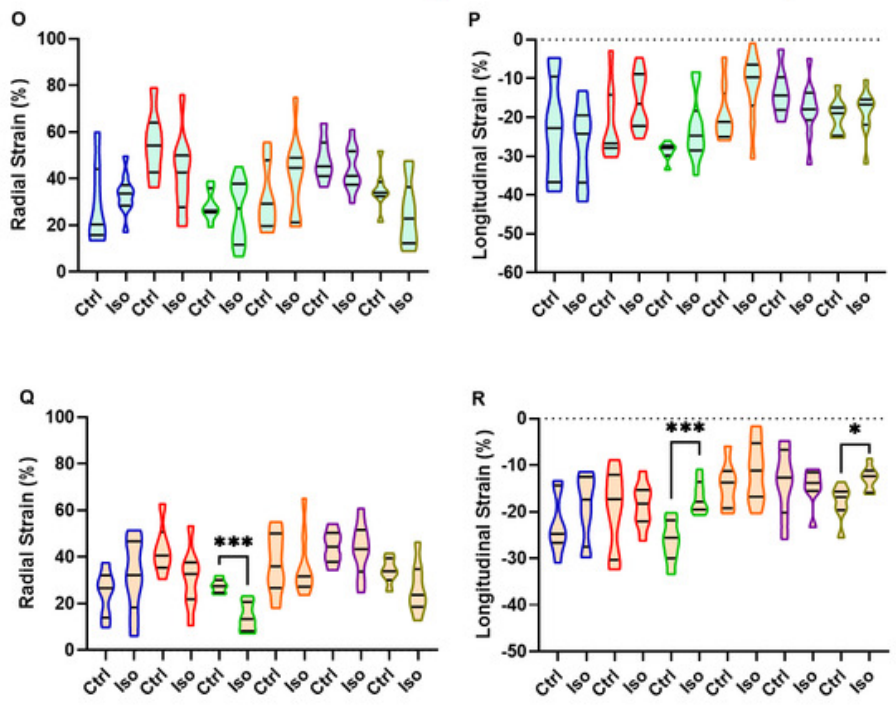